\title{
Light Scattering Studies on Solutions Containing Calcium Phosphates
}

\author{
T. P. FEENSTRA AND P. L. DE BRUYN \\ Van't Hoff Laboratory, Transitorium 3, Padualaan 8, Utrecht, The Netherlands
}

Received March 9, 1979; accepted June 15, 1979

\begin{abstract}
A number of light scattering experiments was performed on calcium and phosphate containing solutions at $\mathrm{pH} 8.33$ and $26^{\circ} \mathrm{C}$. Supplementary information was obtained by means of dynamic light scattering, scanning electron microscopy, and transmission electron microscopy. The measurements prove that during the period in which amorphous calcium phosphate is present, coagulation is the dominant process that gives rise to the observed changes in the scattering intensity.
\end{abstract}

\section{INTRODUCTION}

The formation of hydroxyapatite (HAP), $\mathrm{Ca}_{10}\left(\mathrm{PO}_{4}\right)_{6}(\mathrm{OH})_{2}$ from supersaturated solutions has been studied extensively, largely because of the occurrence, in dental enamel and bones, of a compound which resembles HAP very much. These investigations pointed out that the precipitation of HAP from aqueous solutions is preceded by the formation of a precursor phase commonly referred to as an amorphous calcium phosphate (ACP) (1).

Many attempts have been made to elucidate the nature of ACP. Several authors believed it to be identical in composition to other calcium phosphates, notably brushite (DCPD) (2), octacalcium phosphate (OCP) (3), and tricalcium phosphate (TCP) (6-8). However, more recent work by Termine and Eanes (9) indicated that the chemical composition of ACP is $\mathrm{pH}$-dependent and that at most short-range order is to be expected. They therefore suggested that ACP best be considered a glass-like substance. Lateron Betts and Posner (10) concluded from an X-ray radial distribution study of ACP that this material is better viewed as an amorphous clustering of microcrystallite material than a random network structure.

Other investigations pointed to the exist- ence of OCP as an intermediate in the transformation process which eventually results in the precipitation of HAP (11-13). At low supersaturations the precipitation of HAP has been found to proceed without formation of a precursor phase $(5,14)$.

In this investigation which was primarily undertaken to get more information about the first stages of the HAP precipitation process we have made use of light scattering measurements. The great advantage of this technique is that an in situ study of the system is possible.

\section{EXPERIMENTAL}

Two types (I and II) of experiments were performed. In type I experiments the early stage in the precipitation process was followed by light scattering measurements and in type II experiments the same process was observed by registering the amount of alkali consumed at constant $\mathrm{pH}$ as a function of time.

In both series of experiments the supersaturation was built up by injecting separately a solution containing $0.200 \mathrm{M} \mathrm{Ca}$ $\left(\mathrm{NO}_{3}\right)_{2}$ and a solution of $0.060 \mathrm{M} \mathrm{K} \mathrm{K}_{2} \mathrm{HPO}_{4}$ and $0.060 \mathrm{M} \mathrm{KH}_{2} \mathrm{PO}_{4}$ into 3 liters of a solution either $0.150 M$ (I) or $0.164 M$ (II) in $\mathrm{KNO}_{3}$ and of $\mathrm{pH} 8.33 \pm 0.02$. A detailed description of the apparatus and injection 
procedure is given elsewhere (13). In these experiments reagent-, analytical-, and purissimum-grade (Merck suprapur) $\mathrm{KNO}_{3}$ were used but no significant effect of the purity was noted on the experimental results. All other reagents were of analytical grade and the water was twice distilled. During an experiment nitrogen was bubbled through the solution to prevent uptake of $\mathrm{CO}_{2}$. The system was thermostatted at $26.0 \pm 0.2^{\circ} \mathrm{C}$. The stirring speed was $550 \mathrm{rpm}$. Changing the speed by as much as $250 \mathrm{rpm}$ did not affect the results. The initial concentration of calcium in the supersaturated solution varied between 1.99 and $2.10 \mathrm{mmole} / \mathrm{liter}$ and the total phosphate concentration between 1.20 and $1.37 \mathrm{mmole} / \mathrm{liter}$. The ratio of these concentrations $(\mathrm{Ca} / \mathrm{P})$ attained values between 1.51 and 1.67 .

In the type I experiment the solutions were made as dust-free as possible by filtration through $0.45-\mu \mathrm{m}$ Millipore filters prior to being introduced into the closed reaction vessel which was also made dust-free. The $\mathrm{KNO}_{3}$ concentration in these tests was $0.15 M$. A $0.033 \mathrm{M}$ Tris $-\mathrm{HNO}_{3}$ buffer was present to maintain a pH of $8.33 \pm 0.02$ for $2 \mathrm{hr}$ after injection of the calcium and phosphate solutions. The time required to build up the supersaturation was always less than $10 \mathrm{~min}$. A $10-\mathrm{ml}$ sample was then taken for light scattering measurements in a FICA 50. These measurements were done with unpolarized light at a wavelength of 436 or $546 \mathrm{~nm}$ as a function of time.

Only those experiments which showed no effects due to spurious dust particles were used in the analysis. Quasielastic light scattering measurements (15) with an argon ion laser $(\lambda=514 \mathrm{~nm})$ were done with one sample. With this technique the diffusion coefficient of the scattering particles can be evaluated from the fluctuations in the scattered light intensity due to Brownian motion.

In type II experiments the $\mathrm{pH}$ was kept constant at 8.33 with a Mettler $\mathrm{pH}$-stat by using an Ingold 465-35 combination electrode which was calibrated with Electrofact buf- fer solutions. The $0.025 \mathrm{M} \mathrm{CO}_{2}$-free $\mathrm{KOH}$ solution was injected by the same procedure as used for the calcium and phosphate solutions. To reach the same ionic strength as in type I experiments the $\mathrm{KNO}_{3}$ concentration was $0.164 M$. Samples $(250 \mathrm{ml})$ for solidstate analysis were taken from the reaction vessel. These were rapidly filtered through 100 -nm Millipore filters and subsequently freeze dried. Photographic Debye-Scherrer patterns of the solid material were recorded with an Enraf-Nonius diffractometer using $\mathrm{Cu} K_{\alpha}$ radiation. Scanning electron microscopy was performed in a Cambridge Stereoscan 150. For transmission electron microscopy two sample preparation techniques were used. With the first technique the sample was sprayed on freshly cleaved mica and then a carbon replica was made. In the second method the freeze dried precipitate was redispersed in acetone. A drop of this dispersion was then allowed to dry on a parlodion carbon-coated grid and afterward viewed in a Philips EM 201. Sedimentation experiments were done in a Beckman Spinco (model E) ultracentrifuge.

\section{RESULTS}

The uptake of alkali as a function of time as recorded in a typical type II experiment is displayed in Fig. 1. The parameter $\alpha$ denotes the degree of advancement of the reaction $(0 \leqslant \alpha \leqslant 1)$ based upon the assumption that HAP is the most stable solid phase. The magnitude of $\alpha$ is evaluated by dividing the experimental amount of alkali consumed by the total amount necessary to precipitate HAP and to yield a solution saturated with respect to this compound. In making these calculations the presence of the complexes $\mathrm{CaH}_{2} \mathrm{PO}_{4}^{+}, \mathrm{CaHPO}_{4}^{0}, \mathrm{CaPO}_{4}^{-}$, and $\mathrm{CaOH}^{+}$ in the solution phase was also considered.

A detailed description of the calculation procedures is given elsewhere $(13,16)$. The precipitation curve in Fig. 1 may be divided into three regions ( $\mathrm{A}, \mathrm{B}$, and $\mathrm{C}$ ).

As pointed out previously (13) this division 
correlates with the turbidity curve given by Termine and Eanes (9) and allows one to assign to each region a characteristic feature. In region $\mathrm{A}$ amorphous calcium phosphate is present. Region $\mathrm{B}$ is correlated with the amorphous-crystalline conversion whereas in region $C$ the growth of apatitic crystals takes place. The light scattering data of type I experiments were gathered in region A. Solid samples taken in this region gave a Debye-Scherrer pattern of an amorphous calcium phosphate (17). The solution appeared slightly colloidal. No coarse precipitate could be observed.

All light scattering measurements showed an angular dependence as depicted in Fig. 2 where the reciprocal scattering intensity in arbitrary units is plotted as a function of $\sin ^{2} 1 / 2 \theta$. This observation implies that the angular dependence of the scattered light did not alter with time, i.e., the two curves in Fig. 2 coincide on multiplying the intensity with an appropriate scale factor. The maximum at high angles was noted in all experiments but we are not sure whether it has any real significance. This maximum in Fig. 2 correlates with low I values (ordinate in figure is reciprocal intensity) which could not be measured with the precision possible at higher intensities (lower $\sin ^{2} 1 / 2 \theta$ ). Prob-

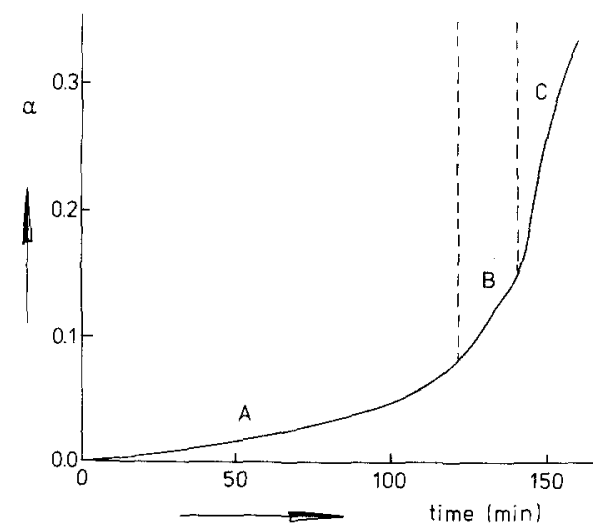

FIG. 1. Alkali uptake curve for a type II experiment: $\mathrm{pH}=8.33, T=26^{\circ} \mathrm{C}, 0.164 M \mathrm{KNO}_{3}$, total calcium $=1.98 \mathrm{mmole} /$ liter, total phosphate $=1.21 \mathrm{mmole} /$ liter.

ably it is due to a systematic error. Figure 3 displays the dissymmetry at $75^{\circ}$, i.e., the ratio of the intensities at $75^{\circ}$ and $105^{\circ}$. We chose the dissymmetry at $75^{\circ}, Z\left(75^{\circ}\right)$, in preference to that at $45^{\circ}$ because both intensities (at 75 and $105^{\circ}$ ) fall on the straight line portion of the $I^{-1}$ vs $\sin ^{2} 1 / 2 \theta$ curve.

The dissymmetry was evaluated at two different wavelengths to determine if the observed fluctuations in dissymmetry could not originate from convection due to small temperature differences between the measuring cuvette and the surroundings. As can

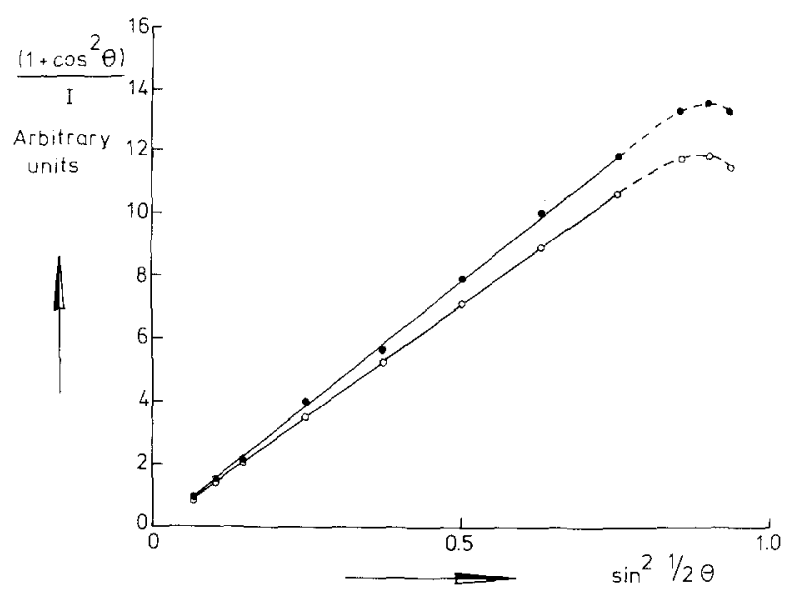

FIG. 2. Reciprocal scattering intensity as a function of $\sin ^{2} 1 / 2 \theta$ at two times for a sample taken in region $A ; \lambda=546 \mathrm{~nm}$. The data are corrected for the scattering of the pure liquid. $t=10 \mathrm{~min}$; $O, t=40 \mathrm{~min}$. 


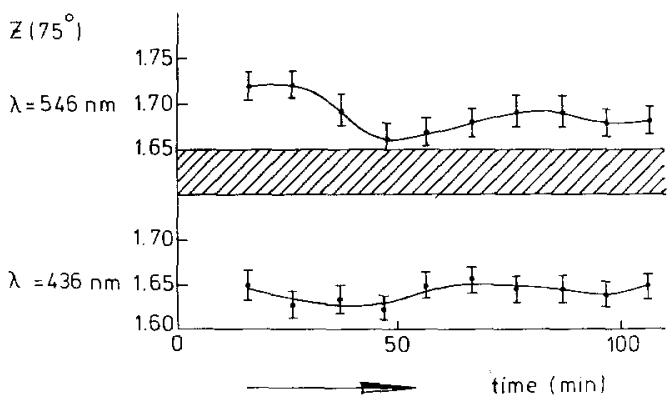

FIG. 3. $Z\left(75^{\circ}\right)$ as a function of time for a sample taken in region $\mathrm{A}$ at two different wavelengths. The data are corrected for the scattering of the pure liquid.

be seen from this figure the fluctuations at $546 \mathrm{~nm}$ are larger than those at $436 \mathrm{~nm}$. This observation leads to the conclusion, as will be amplified in the discussion, that convection is not responsible for the fluctuations.

From the figure we also note that the experimental errors in the evaluated dissymmetry are fairly large but the indicated sinusoidal fluctuations were quite reproducible.

In Fig. 4 the scattering intensity at $75^{\circ}$ and $436 \mathrm{~nm}$ is shown as a function of time. This curve was more difficult to reproduce (shape and height above the zero intensity datum) than those plots in the previous figures. However, in all the experiments the increase in intensity accompanying the progress of the process was found to be small as may be seen in Fig. 4.

The time to reach region $\mathrm{C}$ (Fig. 1) was always shorter in the well-stirred reaction vessel than in the unstirred cuvettes used in the light scattering experiments, while type I and type II experiments in the reaction vessel had the same time scales. This time difference is readily established because the beginning of region $\mathrm{C}$ is characterized by visible precipitation. Ultracentrifugation and transmission electron microscopy measurements on liquid samples taken in region A were not possible because of the low concentration of the colloidal particles formed in this region.

Scanning electron microscopy on solid samples gave no useful information because the particles were too small. Only when the $\mathrm{pH}$ (and thus the supersaturation) was increased to a value of 8.45 could spherical particles identical with those described by Nylen et al. (18) be observed. Transmission electron micrographs of redispersed solid samples revealed circular flat particles of diameter up to $0.5 \mu \mathrm{m}$. These particles displayed the electron lucent holes characteristic of ACP (18). However, we are not sure that we were not perhaps looking at artifacts.

Dynamic light scattering revealed that the apparent diffusion coefficient of the particles in region A decreased from $4.4 \times 10^{-13}$ to $2.3 \times 10^{-13} \mathrm{~m}^{2} / \mathrm{s}$. The fact that the particles are growing during these measurements prevented us from extrapolating the measured values of $D$ to $\sin ^{2} 1 / 2 \theta=0$ to obtain the real diffusion coefficient.

\section{DISCUSSION}

In the interpretation of the light scattering data we shall be guided by two important experimental observations. We noted a small increase in scattering intensity with time (Fig. 4) whereas an increase of the particle radius by about a factor two may be derived from the dynamic light scattering measurements. The latter conclusion fol-

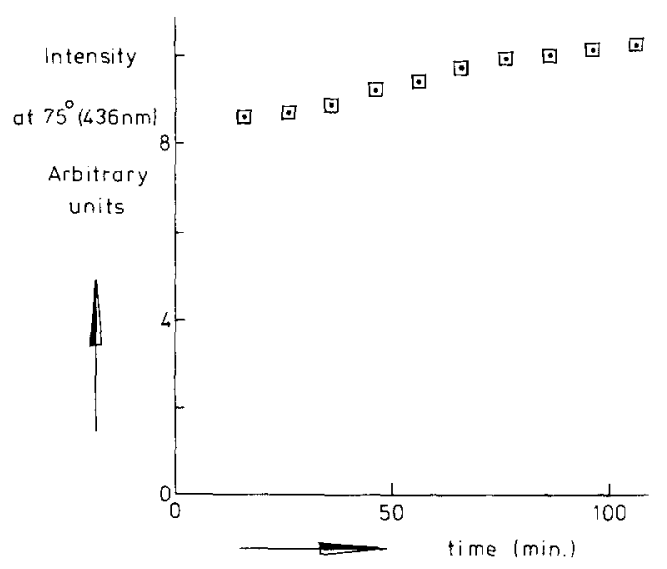

FIG. 4. Intensity as a function of time for the same experiment as in Fig. 3. $\lambda=436 \mathrm{~nm}, \theta=75^{\circ}$. The data are corrected for the scattering of the pure liquid. 
lows from the Einstein relation

$$
D=\frac{k T}{f}
$$

which shows that the diffusion coefficient $(D)$ varies inversely with the friction factor $f$ which in turn is assumed proportional to the particle radius.

Now it is possible to account for both these observations if coagulation is the dominant process that occurs in region $\mathrm{A}$ (see Fig. 1). Furthermore, the observed effect of stirring on the length of region A can then be interpreted as an example of orthokinetic flocculation (19). This suggestion is reasonable in view of the rather large particle diameters (in the order of $1 \mu \mathrm{m}$ ).

The light scattering data should therefore provide us with information about the nature of the coagulates that are being formed in the solution. For a number of reasons, the interpretation of the results is however complicated. First, the dynamic light scattering study indicates the particles to be quite large; one may therefore question whether the Rayleigh-Gans-Debye theory may be applied in the analysis. Second, from studies of Nylen et al. (18) we know that the ACP phase in solution is quite polydisperse. Third, an observed angular dependence of scattering is not uniquely related to one specific particle form.

We now proceed to demonstrate that taking cognizance of these complications, the observed scattering behavior of our system may be accounted for by assuming the coagulate to exist as polydisperse, randomly oriented thin disks.

The particle scattering function for the above mentioned disks is given by Porod (20)

where

$$
P(\theta)=2 / u^{2}\left[1-\left(\frac{1}{u}\right) J_{1}(2 u)\right]
$$

$$
u=\frac{4 \pi a}{\lambda} \sin (\theta / 2)=h a
$$

and where $a$ designates the radius of the disk, $\lambda$ the wavelength in the medium, $\theta$ the angle of observation. $J_{1}(x)$ is a Bessel function of the first kind and of order one.

In the following we shall show that we may indeed apply the Rayleigh-GansDebye theory and thus Eq. [2] to the analysis. Distortion of the wavefront of the light beam may become important if it has to pass through a considerable length of the particle. The fraction of orientations with the disk parallel to the incident light beam is proportional to $\delta / a$, where $\delta$ is the thickness of the particle. For reasonably thin disks this fraction should be small and therefore also the distortion of the wavefront. The effects of interparticle interference and interactions between the particles will be small because of the small weight fraction of the calcium phosphate solid in the beginning of the process (see Fig. 1).

The effect of polydispersity will be considered by introducing a $\log$ normal distribution function because of its frequent encounter in analyses of particle size distributions (21). Such a distribution function as given by the relation (22)

$p(a)=\frac{1}{(2 \pi)^{1 / 2} a \sigma} \exp -\frac{\left(\ln a-\ln a_{\mathrm{m}}\right)^{2}}{2 \sigma^{2}}$

where $\ln a_{\mathrm{m}}$ is the mean value of $\ln a$ and $\sigma$ is the standard deviation of the distribution of $\ln a$.

The dissymmetry at $75^{\circ}$ is then determined by the expression

$$
Z\left(75^{\circ}\right)=\frac{\int_{0}^{\infty} p(a) P\left(75^{\circ}\right) a^{6} d a}{\int_{0}^{\infty} p\left(a^{\prime}\right) P\left(105^{\circ}\right) a^{\prime 6} d a^{\prime}}
$$

In Fig. 5 calculated $Z\left(75^{\circ}\right)$ curves for different values of $\sigma$ are given as a function of $a_{\mathrm{m}}$. On comparing the curves in Fig. 5 with the experimental curves $\left(Z\left(75^{\circ}\right)\right.$ versus time) in Fig. 3, it should be noted that an increase

${ }^{1}$ Here we suppose the thickness of the particles to be proportional to their radius. 


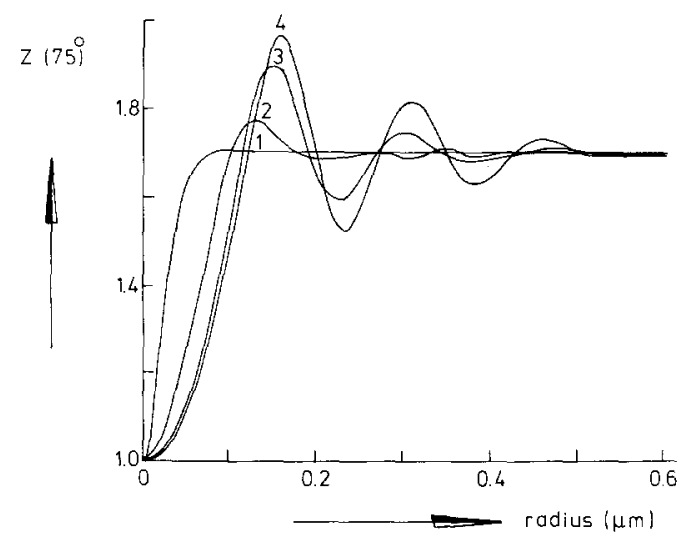

FIG. 5. Calculated $Z\left(75^{\circ}\right)$ as a function! of the radius of a very thin circular disk; $\lambda=546 \mathrm{~nm}, n=1.3344$. Different curves represent different logari hmic normal distributions. (1) $\sigma=0.5$; (2) $\sigma=0.25$; (3) $\sigma=0.10$; (4) $\sigma=0.001$.

in time is equivalent to an increase in mean particle radius. The limiting dissymmetry value at high $a_{\mathrm{m}}$ (see Fig. 5) equals [ $\sin ^{2}$ $\left.\times\left(105^{\circ} / 2\right)\right] /\left[\sin ^{2}\left(75^{\circ} / 2\right)\right]=1.698$ because under this condition the Bessel function in Eq. [2] approaches a value of zero. From Fig. 5 we furthermore note that this limiting value is reached earlier at high values of $\sigma$, i.e., with increased polydispersity. This result implies that the broader the distribution function, the smaller the mean logarithmic particle size for which a plot of $I^{-1}$ as a function of $\sin ^{2}(1 / 2 \theta)$ will approach a straight line (see Fig. 2).

We also calculated the $P(\theta)$ and $Z\left(75^{\circ}\right)$ for randomly oriented thin square platelets. These calculations show a striking resemblance to those for the disk. The detailed form of the platelets is therefore not important to this analysis. According to Eq. [2] $u$ is proportional to $a / \lambda$. The calculated dissymmetry curves should therefore be shifted toward the left hand-side in the diagram (e.g., Fig. 5) at smaller wavelengths. This also implies that if large fluctuations are observed at $546 \mathrm{~nm}$, for example, smaller ones are expected at $436 \mathrm{~nm}$. The experimental results at these two wavelengths (see Fig. 3) are in accordance with our analysis.

At this point in the discussion we should Journal of Colloid and Interface Science, Vol. 73, No. 2, February 1980 mention that the radius of the particles at the start of the precipitation process as estimated from Fig. 5 should lie at most between 0.3 and $0.4 \mu \mathrm{m}$. Insertion of the expression for the friction factor of a sphere in Eq. [1] yields a radius of about $0.5 \mu \mathrm{m}$ for the particles at the beginning of region $\mathrm{A}$ when using the measured diffusion coefficients. However, as the friction factor of thin circular plates is about two-thirds of that of a sphere of the same radius, the radius calculated from the measured diffusion coefficients could even be as high as $0.75 \mu \mathrm{m}$.

The above calculations do, however, not prove that the coagulates have a platelike habit. In fact Nylen et al. (18) showed the primary particle form for ACP to be that of a sphere. The radius of the ACP spherules was observed to range from 100 to $600 \AA$. The same authors noticed that these spheres coagulate into branched chainlike structures. In the light of these experimental facts we can only conclude that the flocs mimic disks in their light scattering properties. This means that there is some resemblance between the characteristic function for disks and the characteristic function of the coagulates. This characteristic function was introduced by Porod (23) and can be represented by the following relation:

$$
\gamma_{0}(r)=\vec{V}(\mathbf{r}) / V
$$

where $V$ represents a volume and $V(\mathbf{r})$ is a volume common to the particle itself and the particle when it is displaced over a vector $\mathbf{r}$. One can also say that $\gamma_{0}(r)$ represents the probability that a point at a distance $r$ in an arbitrary direction from a given point in the particle will itself also be in the particle. An interesting feature in calcium phosphate systems is the fact that after the conversion of ACP, thus after region B, Eanes and Meyer (11) found lamellar particles.

Eanes et al. (25) also observed in dried samples disk-like ACP particles but considered them to be artifacts. It is possible that the formation of branching chains as was found by Nylen et al. (18) represents 
a form of oriented flocculation which plays a role in the conversion process.

Now oriented flocculation may occur if due to the anisotropy of the primary particles the strength of the interaction varies with the orientation of one particle with respect to another (26). This explanation is hardly to be expected to apply in the case for ACP. A second alternative is oriented flocculation as a result of differences in interaction in different directions between a secondary and a primary particle (27). Both theoretical considerations and experimental facts indicate that in the latter case oriented flocculation leads to chain formation.

\section{CONCLUSIONS}

In the early stages of the precipitation of HAP from solutions supersaturated in calcium and phosphate a number of investigators have shown ACP to be present. From light scattering measurements performed on these solutions it may be concluded that the dominant process at this stage is coagulation of ACP particles. An analysis of the light scattering data suggests a resemblance between the characteristic function for the coagulates and the characteristic function for thin randomly oriented disks. This conclusion is of importance for the elucidation of the conversion mechanism of ACP to OCP as an intermediate phase in the formation of HAP. OCP is generally known to have a platelike habit $(24,28)$.

\section{ACKNOWLEDGMEN'TS}

The authors wish to thank Mr. H. Mos for performing the dynamic light scattering measurements, and Mr. J. Rijnbout for helpful and stimulating discussions. We also wish to acknowledge one of the referees for pointing out that the $\mathrm{ACP}$ system may mimic plate-like particles in its light scattering behavior.

\section{REFERENCES}

1. Eanes, E. D., Gillessen, I. H., and Posner, A. S., Nature (London) 208, 365 (1965).
2. Francis, M. D., and Webb, N. C., Calc. Tiss. Res. 6, 335 (1971).

3. Newesely, H., Arch. Oral Biol., Spec. Suppl. 6, 174 (1961).

4. Füredi-Milhofer, H., Purgarić, B., Brečević, Lj., and Pavković, N., Calc. Tiss. Res. 8, 142 (1971).

5. Nancollas, G. H., and Tomažić, B., J. Phys. Chem. 78, 2218 (1974).

6. Walton, A. G., Bodin, W. J., Füredi, H., and Schwartz, A., Canad.J. Chem. 45, 2695 (1967).

7. Eanes, E. D., and Posner, A. S., Calc. Tiss. Res. 2, 38 (1968).

8. Posner, A. S., Physiol. Rev. 49, 760 (1969).

9. Termine, J. D., and Eanes, E. D., Calc. Tiss. Res. 10, 171 (1972).

10. Betts, F., and Posner, A. S., Mat. Res. Bull. 9, 353 (1974).

11. Eanes, E. D., and Meyer, J. L., Calc. Tiss. Res. 23, 259 (1977).

12. Meyer, J. L., and Eanes, E. D., Calc. Tiss. Res. 25, 209 (1978).

13. Feenstra, T. P., and de Bruyn, P. L., J. Phys. Chem. 83, 475 (1979).

14. Boskey, A. L., and Posner, A. S., J. Phys. Chem. 80, 40 (1976).

15. Berne, B. J., and Pecora, R., "Dynamic Light Scattering with Applications to Chemistry, Biology and Physics." Wiley, New York, 1976.

16. Feenstra, T. P., J. Chem. Ed. 56, 104 (1979).

17. Eanes, E. D., and Posner, A. S., Trans. N. Y. Acad. Sci. 28, 233 (1965).

18. Nylen, M. U., Eanes, E. D., and Termine, J. D., Calc. Tiss. Res. 9, 95 (1972).

19. Kruyt, H. R., "Colloid Science," Vol. I, Chap. VII, p. 291. Elsevier, Amsterdam, 1952.

20. Porod, G., Acta Phys. Austriaca 2, 255 (1949).

21. Jelinek, Z. K., "Particle Size Analysis," 1st ed., p. 14. Wiley, London, 1974.

22. Espenscheid, W. F., Kerker, M., and Matijević, E., J. Phys. Chem. 68, 3093 (1964).

23. Porod, G., Kolloid-Z. 124, 83 (1951).

24. Füredi-Milhofer, H., Brečević, Lj., Oljica, E., Purgarić, B., Gass, Z., and Perović, G., in "Particle Growth in Suspension" (A. L. Smith, Ed.), p. 109. Academic Press, London, 1973.

25. Eanes, E. D., Termine, J. D., and Nylen, M. U., Calc. Tiss. Res. 12, 143 (1973).

26. Jonker, G. H., Thesis, Utrecht, 1943.

27. Thomas, I. L., and McCorkle, K. H., J. Colloid Interface Sci. 36, 110 (1971).

28. Brown, W. E., Smith, J. P., Lehr, J. R., and Frazier, A. W., Nature (London) 196, 1048 (1962). 